\title{
Catatonia in children and adolescents: A case report of a 13 year old child
}

\author{
U Attygalle, TK Athukorala, M RS Jayasekara
}

\section{Abstract}

Catatonia is a syndrome involving abnormalities of movements and position, manifesting as excessive and purposeless motor activity or immobility. Even though this condition is well described in adults, catatonia has been less identified and studied in children and adolescents. Early identification of catatonia can lead to better treatment outcomes in this population. In this case report, we discuss the case of a boy aged 12 years and 11 months, who presented with catatonia, and who was later diagnosed as having early onset schizophrenia.

SLJ Psychiatry 2018; 9(1): 26-28

\section{Introduction}

Catatonia is a syndrome involving abnormalities of movements and position, manifesting as excessive and purposeless motor activity or immobility (1). Extreme negativism, mutism, echopraxia, echolalia and waxy flexibility are also seen. This condition is usually associated with psychiatric disorders such as schizophrenia. However, it can also be seen in medical conditions and neurological conditions (1). As such, the American Psychiatric Association's Diagnostic and Statistical Manual, fifth edition (DSM -5) categorises catatonia into 3 groups - catatonia associated with another mental disorder, catatonia due to another medical condition or unspecific catatonia (2).

Even though this condition is well described in adults, catatonia has been less identified and studied in children and adolescents (1). Early identification of catatonia can lead to better treatment outcomes in this population (3).

Although older studies suggest that catatonia occurs infrequently in children and adolescents, more recent studies show that the prevalence rate may be greater than once thought (4). One study reported the five-year prevalence of catatonia in this age group receiving psychiatric treatment to be $17.8 \%$ (1). A recent crosscultural comparative study in India and Wales indicated that the prevalence of catatonia was $13.5 \%$ in India versus $9.6 \%$ in Wales, among adolescents (5).

In Sri Lanka there are no well-described reports of catatonia in children. However, a case series in 2015 described two adolescents aged 16 and 17 years, both who were found to have medical issues leading to catatonia (6).
In this case report, we describe the case of a boy aged 12 years and 11 months, who presented with catatonia, and who was later diagnosed as having early onset schizophrenia.

\section{Case Report}

The patient was a boy aged 12 years, 11 months. This boy was first referred to our service following changes in behavior, noticed over the preceding week. He was talking on his own without any apparent reason and was noted to be uncharacteristically impulsive and irritable. Some deterioration in academic performances had also seen during the past year. Pre-morbidly, he had an anxious temperament and had few friends. There was a family history of psychiatric illness - a paternal aunt had developed bipolar affective disorder in her early 20's. On mental state examination he was perplexed.

These changes in his behaviour had affected his day to day functioning, and his family found it difficulty to manage his changed behavior at home. Given these changes, and as early onset psychosis was suspected, he was started on treatment with aripiprazole. The dose was gradually increased up to $7.5 \mathrm{mg}$ daily. He improved and returned to his daily routine, including schooling. However, the family defaulted follow up.

During his second presentation five months later, the child had developed unusual fears, irritability and inability to fall asleep. He was socially withdrawn and had not attended school. Mental state examination revealed delusions of control and persecutory delusions. While under observation, he developed catatonic symptoms including waxy flexibility, a staring gaze, mild rigidity, withdrawal, ambitendency and mutism. 
No specific symptoms of mania or depression were noted. Any possibilities of substance abuse or other medical illnesses were excluded through liaison with the pediatric team. His neurological examination, electroencephalography(EEG), blood and urine investigations, including serum electrolytes, urine full report, liver function tests, renal function tests, $\mathrm{C}$ reactive protein, and full blood count were all normal. Other investigations including a video EEG and a computed tomograph (CT) scan of the brain also did not show any abnormalities.

His Bush-Francis Catatonia Rating Scale (BFCRS) score was 14 out of 23. The Positive and Negative Syndrome Scale score (PANSS) was 23/49 in the positive scale; $12 /$ 49 in the negative scale and 42/112 in the general psychopathology scale.

As he showed clear features of catatonia, he was started on a benzodiazepine, clonazepam. This was increased to a $0.5 \mathrm{mg}$ twice daily, at which dose he started to rapidly improve. After 4 days the clonazepam was gradually tapered off. Thereafter he was restarted on aripiprazole, since he continued to have psychotic symptoms and as there was a history of treatment response with this medication. He was later discharged with a working diagnosis of early onset catatonic schizophrenia. On discharge from the hospital he did not have any catatonic features, but continued to have some persecutory ideas, anxieties and social withdrawal.

On follow up the aripiprazole was gradually increased to $15 \mathrm{mg}$ daily. He started to get back to his daily routine and restarted going to school. One month after discharge the score on the BFCRS was 00 with no catatonic symptoms, and the PANSS score was $07 / 49$ in the positive scale; $13 / 49$ in the negative scale and 18/112 in the general psychopathology scale.

Currently the major concern with regards to his management and prognosis is the improvement of negative symptoms of schizophrenia.

\section{Discussion}

Although catatonia is more commonly found among patients with schizophrenia, it is also seen in patients with mania, depression and systemic medical illnesses (7). The presentation and the course of symptoms in this patient, suggest early onset schizophrenia. While the literature suggests that agitated forms of catatonia, accompanied by aggression, are the common presentations of catatonia in younger age groups, our patient exhibited the classic features of catatonia, such as waxy flexibility, rigidity, and catalepsy (1).

The absence of the classic symptoms of catatonia, and the lack of familiarity with more non-specific presentations, may be a reason why catatonia is under recognized among children and adolescents (1).
However, as in our patient, more recent literature suggests that the presentation of catatonia in this age group may be similar to that seen in adult populations (1). Similarly, with regards to psychosis, while agitation, delusions and hallucination are well described symptoms of early psychosis, catatonia as a presenting symptom is rarely described (2).

The literature suggests that acute relief from catatonia can be achieved by benzodiazepines or with electro convulsive therapy (7). In this case, clonazepam was used due to availability, although most reports suggest lorazepam as the first line of treatment in this age group (8). Clonazepam too has been reported in some instances being used successfully to treat catatonia in adults (9). Some studies suggest that antipsychotics can increase catatonic symptoms if used in the acute stage (2). However, others suggest that antipsychotics such as aripiprazole may be effective in the treatment of both catatonia and psychosis. There is also evidence for the use of other second generation antipsychotics such as olanzapine and quetiapine in this age group $(2,10)$.

\section{Acknowledgements}

We would like to acknowledge the support provided by the patient and his parents towards writing this case report.

\section{Disclosure statement}

None declared

U Attygalle, TKAthukorala, M R Sahangee Jayasekara, Child and Adolescent Psychiatry Unit, Teaching Hospital Karapitiya, Galle.

\section{Corresponding author: U Attygalle}

Email: udenaa@gmail.com

http://orcid.org/0000-0002-8064-2817

\section{References}

1. Ghaziuddin N, Dhossche D, Marcotte K. Retrospective chart review of catatonia in child and adolescent psychiatric patients. Acta Psychiatr Scand 2012; 125(1): 33-8.

2. Yoshimura B, Hirota T, Takaki M, Kishi Y. Is quetiapine suitable for treatment of acute schizophrenia with catatonic stupor? A case series of 39 patients. Journal of Neuropsychiatr Dis Treat 2013; 9: 1565-71.

3. Roberto AJ, Pinnaka S, Mohan A, Yoon H, Lapidus KAB. Adolescent Catatonia Successfully Treated with Lorazepam and Aripiprazole. Case Rep Psychiatry 2014; 2014: 309517. 
4. Cohen D, Flament M, Dubos P, Basquin M. Case series: catatonic syndrome in young people. J Am Acad Child Adolesc Psychiatry 1999; 38(8): 1040-6.

5. Chalasani P, Healy D, Morris R. Presentation and frequency of catatonia in new admissions to two acute psychiatric admission units in India and Wales. Psychol Med 2005; 35(11): 1667-75.

6. Isuru LLA, Kuruppuarachchi KALA. A case series of catatonia: Lessons to learn. SL J Psychiatry 2015; 6(1): 29-31.

7. Fink M. Catatonia: a syndrome appears, disappears, and is rediscovered. Can J Psychiatry 2009; 54(7): 437-45.
8. Rosebush PI, Hildebrand AM, Furlong BG, Mazurek MF. Catatonic syndrome in a general psychiatric inpatient population: frequency, clinical presentation, and response to lorazepam. J Clin Psychiatry 1990; 51(9): 357-62.

9. Kumar R. Acute severe catatonia in a young woman with chronic schizophrenia responding to parental clonazepam. Aust N Z J Psychiatry 2001; 35(3): 391.

10. Ceylan MF, Kul M, Kultur SEC, Kilincaslan A. Major depression with catatonic features in a child remitted with olanzapine. J Child Adolesc Psychopharmacol 2010; 20(3): 225-7. 